\title{
Rehabilitation in Hip Dislocation with Bipolar Hemiarthroplasty in Geriatric Patient - A Case Report
}

\author{
Namrata Rathi $^{1}$, Tushar Ubare ${ }^{2}$, Shilpa Khurana ${ }^{2}$, Amrita Sakharwade ${ }^{3}$ \\ ${ }^{1}$ Professor, Department of Neurosciences Physiotherapy, Shri K. R. Pandav College of Physiotherapy, \\ Bhilewada, Bhandara, India. \\ ${ }^{2}$ Professor, Shri K. R. Pandav College of Physiotherapy, Bhilewada, Bhandara, India. \\ ${ }^{3}$ Assistant Professor, Smt. Radhikatai Pandav College of Physiotherapy, Nandanvan, Nagpur.
}

Corresponding Author: Namrata Rathi

DOI: https://doi.org/10.52403/ijhsr.20220314

\begin{abstract}
Hip fracture is the most common injury in the elderly; the rate of occurrence rises exponentially with age, and the aging of the population will ensure that hip fracture remains a major clinical challenge and public health problem in the future. Hip fractures are still the most common cause of morbidity and mortality in the elderly. Hip fractures increase in frequency as people get older. Even though bipolar hemiarthroplasty of the hip is a popular technique, little is known regarding the frequency and treatment of postoperative dislocation. This study looked at 1,934 hips that had been treated with primary bipolar hemiarthroplasty. In older patients with displaced femoral neck fractures, bipolar hemiarthroplasty has also been used to treat osteoarthritis, inflammatory arthritis, and osteonecrosis. Although dislocation after primary bipolar hemiarthroplasty is infrequent, it is linked to a high likelihood of recurrence. Intertrochanteric femur fractures are common in the elderly. Because of increased life expectancy and osteoporosis, their prevalence has risen. Patients are encouraged to move and exercise the affected limbs soon after hip arthroplasty, minimizing bed rest and the rate of problems. THA is a more complicated and expensive procedure than bipolar hemiarthroplasty (BA). Internal fixation is emphasized, as well as early mobilization. For the treatment of sub-capital femoral fractures, hip hemiarthroplasty is a common procedure.
\end{abstract}

Keywords- Hip dislocation, Bipolar hemiarthroplasty, Geriatric population, physical rehabilitation.

\section{INTRODUCTION}

Intertrochanteric fracture is a common condition among the elderly, and it is growing more common as the number of the elderly population grows. An unstable intertrochanteric fracture in the elderly is connected to a significant risk of death of up to $20 \%$ in the first year after surgery. (1). Internal fixation of an unstable intertrochanteric fracture in an elderly patient with osteoporotic bone causes severe collapse, loss of fixation, and lag screw cutout, leading to poor function. (1). Internal fixation can be used to treat the majority of intertrochanteric hip fractures (2). Dynamic devices, also known as a sliding screw/side plate, telescoping nail, sliding nail, dynamic hip screw, and sliding hip screw, are now widely used as reliable methods of internal fixation, even though the operative technique is not always easy and postoperative regimens cannot be standardized. (3). Despite reports of $100 \%$ union rates in patients with well-reduced, stable fractures treated with excellent implant placement, failure rates in elderly patients with unstable fractures, comminution, suboptimal fracture fixation, 
or poor bone quality have reached up to $56 \% .(1)$. In recent decades, the world population's survival has risen globally. This rise in longevity associated with today's more active elderly lifestyle and the chronicity present in this demographic, such as loss incoordination, muscle strength, reflexes, and bone mineral density, results in osteopenia and osteoporosis, has led to ria sing in cases of trauma and thus fractures in the elderly population (4). In older adults, these fractures are the primary cause of injury, physical impairment, and death (5). The risk of fracture can be increased by technical factors during surgery. The effects of the aging population worldwide were linked to the increase in the total number of patients with hip fractures from 1.7 million per year in 1990 to 6.3 million per year in 2050, as well as the rise in socio-economic costs (6). Therefore, the purpose of care for these patients is to retain their functions, mobility, and their activities. (5). Patients with hip fractures may be unable to regain independence due to severe lower extremity edema and decreased edema strength of knee extension, especially after surgery. (7). Low bone mineral density (BMD) osteoporosis puts elderly people at high risk of developing fractures (8). Specific reasons for the successful treatment of these elderly patients focus on patient comorbidities, specific clinical process management, and more or less early postoperative recovery. For patients lying in bed after surgery, the form of fracture, surgery, special care, early active recovery improves the functional outcome in the short term (9). In postoperative treatment for older people, improvement in the standard of care and recovery with an emphasis on fall prevention based on these outcomes should be introduced (5). The patient advanced from NWB walking with a walker with limited support to NWB walking with a walker without assistance to purely supervised walking with a walker. (10). At two years, 85 percent of patients were able to walk independently or with just one cane. Patients are allowed to bear their full weight right after implantation, allowing for maximum mobilization and rehabilitation (10). Bipolar hemiarthroplasty is appealing in patients with neurologic impairment and risk of instability, such as those who have had a stroke or who have Parkinson's disease or dementia (10).

\section{PATIENT INFORMATION}

A 76-year-old male was brought to casualty by relatives with chief complaints of pain and swelling over the left hip region. The patient also had dressing over the left hip region. The patient gives an alleged history of RTA on $25 / 10 / 21$ at his local place when he was hit by a two-wheeler sustaining injury over the left hip joint. The patient was then admitted to a private hospital and operated on $25 / 10 / 2021$ for an Intertrochanteric femur fracture left side with DHS plate. 4 weeks post-operative patient had an imbalance in walking in a walker and felt again sustaining injury over left hip when he was again admitted and operated on in the same hospital on $13 / 12 / 2021$ for refracture with DHS failure and managed with bipolar hemiarthroplasty. On postoperative day 2, while doing dressing in a lateral position patient felt a sudden click sound followed by pain over his left hip joint. The pain was followed by discharge from sutures over the left hip.

CLINICAL FINDINGS: The patient in the supine position was examined with both shoulders at the same level with the left lower limb in external rotation and lateral border of the foot touching the bed. Vital signs were normal on physical examination, including temperature, pulse rate of 90 beats per minute, respiratory rate of 24 breaths per minute, and blood pressure of $160 / 100 m m h$. The patient is a known case of HTN on medication Telma 40 once a day. Swelling is present over the left hip region. No sinuses present. Muscle wasting is seen over the hip and thigh. Multiple grades 1 bedsore of size $2 * 2 \mathrm{~cm}$ present over buttocks. Tenderness is present over the greater trochanter and anterior joint line 
over the left side. Shortening of $4 \mathrm{~cm}$ present over the left lower limb. Hip range of motion could not be done. Knee range of motion unable to flex due to pain. Active ankle and toe movements are present. Upon physical examination, it was determined that he was febrile.

\section{Operative Notes}

The patient has undergone Implant removal infected bipolar prosthesis from the left hip. Under spinal anesthesia, the patient has taken an OT table in the right lateral position. Stapler removal is done from the left hip over the previous incision. Softtissue dissection was done and pieces of dead tissue were removed. Bipolar Prosthesis removed, pus collection around the hip joint is sent for culture and sensitivity.

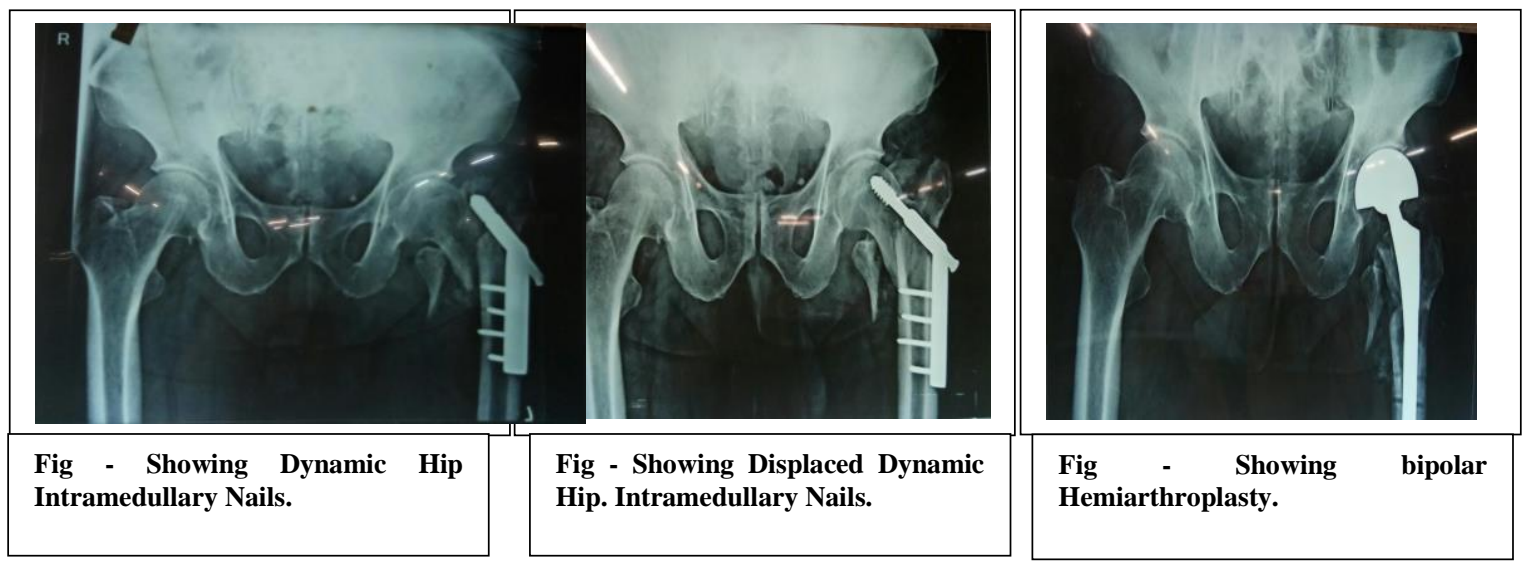

\section{THERAPEUTIC INTERVENTION:}

Our short-term goal is to improve muscle strength, reduce edema, improve posture while sitting, minimize secondary complications such as the increased ability to relax, enhance functional abilities, increase independence and prevent disability.

Long term goal was to do not allow symptoms to re-occur, improve the quality of life of the patient and make his return to functional activities. To restore muscle strength, endurance, and function.

The patient received injections of INJ Ceftriaxone, INJ Amikacin, INJ Tramadol, TAB Dolo, TAB Pan 40, TAB Calcium, TAB Limcee twice a day.

A skilled orthopedic physiotherapist provided regular physiotherapy to the patient in the orthopedic inpatient ward for 15 days. To improve independent walker walking with non-weight bearing and minimal assistance for remembering daily activities, the sessions were initiated. The focus of treatment and recovery should be on regaining physical capacity and avoiding complications. For left thigh pain, cryotherapy with ice packs was used to manage pain and muscle spasms, and elevation with a pillow was used to position the left lower limb. We also offer rehabilitation exercises such as ten-second holds for ten repetitions of isometric contractions for the left hamstrings, quadriceps, and gluteus muscles. For left hip flexion-extension, abduction-adduction, and ankle-toe movements, ten repetitions of active assisted movements were performed. The patient was told to get out with assistance and take a few steps with help (walking frame). Mobilization continued with more walking exercise. Patients were encouraged to get out of bed and use the restroom on their own, as well as walk safely on flat surfaces and stairs with crutches. The purpose of the physiotherapy sessions was to keep the lower left limb muscle intact while strengthening the lower right limb and both upper limbs to enable independent walker walking with non- 
weight bearing and limited assistance for everyday activities. With suitable safety precautions, progressive active resistive workouts utilizing weights for the upper limbs and a weight cuff for the right lower limb were used. Family members were taught and instructed on how to follow a written protocol for carrying out the home exercise program.

\section{RESULTS}

The ability to initiate active-assisted to active hip flexion-extension and abduction-adduction motions, as well as independence in daily living tasks with minimal assistance or supervision, improved. The patient advanced from NWB walking with a walker and minimal help to NWB walking with a walker and simply being supervised.

\section{DISCUSSION}

The study shows that patients can take a few months to regain normal muscle function after undergoing surgery (1). Respiratory disorders and other issues inherent in lack of mobility can be avoided by therapists, although this is often not possible because of the general state of health of the patient. Using the weightbearing exercise of the lower limb improves the functional outcome measure (3). Physical therapy helps to increase muscle strength and improve the protection and performance of walking, thereby enabling the elderly to become more stable (5).

\section{CONCLUSION}

The above studies suggest that early rehabilitation of physiotherapy contributed to the improvement of the patient's functional objectives, which are a significant understanding of a good recovery.

Author's Contribution: For the concept, assessment, evaluation, data collection analysis, and interpretation of the data, each author made the best contribution.
Informed Consent: Proper consent was taken from the patient's son for writing the case report.

Funding: There are no financial conflicts of interest to disclose.

Conflict Of Interest: Authors have no conflicts of interest to disclose.

\section{Acknowledgement: None}

\section{REFERENCES}

1. Sinno K, Sakr M, Girard J, Khatib H. The effectiveness of primary bipolar arthroplasty in the treatment of unstable intertrochanteric fractures in elderly patients. North Am J Med Sci. 2010;561-8.

2. Fan L, Dang $X$, Wang K. Comparison between Bipolar Hemiarthroplasty and Total Hip Arthroplasty for Unstable Intertrochanteric Fractures in Elderly Osteoporotic Patients. Zhang C, editor. PLoS ONE. 2012 Jun 22;7(6):e39531.

3. Kandel L, Hernandez M, Safran O, Schwartz I, Liebergall M, Mattan Y. Bipolar hip hemiarthroplasty in a patient with an above-knee amputation: a case report. J Orthop Surg. 2009 Dec;4(1):30.

4. Bűcs G, Dandé Á, Patczai B, Sebestyén A, Almási R, Nöt LG, et al. Bipolar hemiarthroplasty for the treatment of femoral neck fractures with the minimally invasive anterior approach in elderly. Injury. 2021 Mar;52:S37-43.

5. Purushe d. Physiotherapy rehabilitation in elderly patients with a postoperative femoral neck fracture. J med pharm allied sci. 2021 nov $15 ; 10(6): 3883-5$.

6. Tunioi ZH, Jhatiyal RA, Akhund MA, Abbasi MK, Maheshwari LD, Memon AR. Functional Outcome of Intracapsular Femoral Neck Fractures Treated by Bipolar Hemiarthroplasty. Pak J Med Health Sci. 2021 May 30;15(5):988-91.

7. Gu G, Wang G, Sun D, Qin D, Zhang W. Cemented bipolar hemiarthroplasty with a novel cerclage cable technique for unstable intertrochanteric hip fractures in senile patients. Chin J Traumatol Engl Ed. 2008 Feb;11(1):13-7.

8. Shah SN, Wainess RM, Karunakar MA. Hemiarthroplasty for Femoral Neck 

report.

Fracture in the Elderly. J Arthroplasty. 2005 Jun;20(4):503-8.

9. Kayali C, Agus H, Ozluk S, Sanli C. Treatment for Unstable Intertrochanteric Fractures in Elderly Patients: Internal Fixation versus Cone Hemiarthroplasty. J Orthop Surg. 2006 Dec;14(3):240-4.

10. Iorio R, Healy WL, Lemos DW, Appleby D, Lucchesi CA, Saleh KJ. Displaced Femoral
Neck Fractures in the Elderly. Clin Orthop. 2001;(383):14.

How to cite this article: Rathi N, Ubare T, Khurana $S$ et.al. Rehabilitation in hip dislocation with bipolar hemiarthroplasty in geriatric patient- a case report. Int J Health Sci Res. 2022; 12(3): 114-118. DOI: https:// doi.org/10.52403/ijhsr.20220314 